University of Nebraska - Lincoln

DigitalCommons@University of Nebraska - Lincoln

July 2002

\title{
A model for mechanochemical transformations: Applications to molecular hardness, instabilities, and shock initiation of reaction
}

Tadeusz Luty

University of Nebraska - Lincoln

Piotr Ordon

Institute of Physical and Theoretical Chemistry, Technical University of Wrocław, Poland

Craig J. Eckhardt

University of Nebraska - Lincoln, ceckhardt1@unl.edu

Follow this and additional works at: https://digitalcommons.unl.edu/chemistryeckhardt

Part of the Chemistry Commons

Luty, Tadeusz; Ordon, Piotr; and Eckhardt, Craig J., "A model for mechanochemical transformations: Applications to molecular hardness, instabilities, and shock initiation of reaction" (2002). Craig J. Eckhardt Publications. 4.

https://digitalcommons.unl.edu/chemistryeckhardt/4

This Article is brought to you for free and open access by the Published Research - Department of Chemistry at DigitalCommons@University of Nebraska - Lincoln. It has been accepted for inclusion in Craig J. Eckhardt Publications by an authorized administrator of DigitalCommons@University of Nebraska - Lincoln. 


\title{
A model for mechanochemical transformations: Applications to molecular hardness, instabilities, and shock initiation of reaction
}

\author{
Tadeusz Luty \\ Department of Chemistry, University of Nebraska-Lincoln, Lincoln, Nebraska 68588-0304 \\ and Institute of Physical and Theoretical Chemistry, Technical University of Wroctaw, 50-370 Wroctaw, \\ Poland \\ Piotr Ordon \\ Institute of Physical and Theoretical Chemistry, Technical University of Wrocław, 50-370 Wrocław, Poland \\ Craig J. Eckhardta) \\ Department of Chemistry, University of Nebraska-Lincoln, Lincoln, Nebraska 68588-0304
}

(Received 3 May 2001; accepted 24 April 2002)

\begin{abstract}
A basic theoretical structure for mechanochemical transformations based on prior models for solid-state reactions and HOMO-LUMO (highest occupied molecular orbital-lowest unoccupied molecular orbital) gap closing produces the concept of distortion-induced molecular electronic degeneracy (DIMED) of the highest occupied and lowest unoccupied molecular orbitals of an energetic molecule. Both intermolecular and intramolecular charge transfer are involved. The resulting distortion-induced local instability, a mechanochemical effect, leads to chemical transformations and can be analyzed by renormalization of the molecular hardness through the molecular deformation energy. Linear combinations of normal modes are shown to be useful for description of the mechanically induced reaction path. Numerical calculations for the RDX (hexahydro-1,3,5-trinitro-1,3,5-triazine) molecule are used to construct a path for initiation of a reaction by shock. They show the breaking of a single $\mathrm{N}-\mathrm{N}$ bond as the primary step. DIMED is shown to be a kind of "inverse Jahn-Teller effect" leading to the general conclusion that distortion-induced instabilities and mechanically induced reactions require some, but not necessarily complete, HOMO-LUMO gap closure. This indicates that large local strains due to defects or cracks will contribute to DIMED. The DIMED concept, because of its generality, has wide applicability in solid-state chemistry. (C) 2002 American Institute of Physics.
\end{abstract}

[DOI: $10.1063 / 1.1485968]$

\section{INTRODUCTION}

The role of mechanical energy in the chemistry of molecular systems is of growing importance. Although microscopic models exist that describe the role of thermal, electrical and photonic fields on chemical reactions, a general model for mechanical fields has not yet been formulated. Aside from the obvious relevance to solid-state reactions and phase transitions, many important applications remain: strength of materials, detonation, conformational polymorphism, ambient-gas independent triboluminescence, laserpulse-generated shock waves and sonochemistry in general, as well as the configuration and folding of macromolecules under stress. Although chemists often speak of the strain a molecule undergoes, it is rarely in the context of the molecule's response to an external field. Indeed, a general microscopic model for the many kinds of mechanochemical transformations has not been available. The breadth of these applications in chemistry warrants development of such a conceptual framework. This paper reports such a model and applies it specifically to the problem of shock initiation of a molecular decomposition although, as noted, it may be ex-

${ }^{a}$ Electronic mail: eckhardt@unlserve.unl.edu tended to any problem where a transformation on the molecular level is caused by an applied mechanical field. The application to shock initiation of a reaction is particularly relevant to the behavior of energetic materials.

In spite of centuries of use, explosives present many scientific problems. Chief among these is the mechanism of detonation. The enormous significance of energetic materials lends significant impetus to understanding detonation, particularly in the case of secondary explosives where both stability and high power are desirable. In recent years, there has been much work done in the field and within the last decade new models have been proposed. As Fried et al. ${ }^{1}$ have observed, "Understanding the first step in the response of energetic molecules to shock is a topic of considerable importance because it ultimately would shed light on the sensitivity properties of energetic materials." It is the objective here to provide a theoretical framework upon which understanding of that initial step can be built.

The problem of detonation or any sudden input of mechanical energy is complicated by the large span of time and length scales involved. This range is on timescales shorter than and up to those characteristic of molecular vibrations. Such a front is far from thermal equilibrium and initiation of decomposition at the front has to be treated as a nonergodic, 
nonequilibrium process. The first step in a detonation involves the initial effect of the shock wave on a molecule and does not necessarily involve complete decomposition of the molecule but rather the initial response that may introduce the mechanism of decomposition. The initial response at such a front appears to be an athermal transformation where the mechanical energy of the shock does not have to be converted into heat to become available as activation (excitation) energy. Thus, a comprehensive theory of detonation must examine the first step mechanism of energy transfer from the shock wave into the molecular degrees of freedom.

Reviews of detonation mechanisms proposed for explosive molecular crystals have been recently published. ${ }^{1,2}$ Shock compression of an energetic material is well characterized by translational overheating (phonon quasitemperature). In a model proposed by Dlott and Fayer, ${ }^{3}$ it is assumed that there is continuous energy flow from the compressively heated phonon bath into vibrational doorway modes mediated by a multiphonon up-pumping mechanism that heats the molecules to temperatures at which chemical bonds break. This mechanism requires times of the order of hundreds of picoseconds for a ladder of vibrational states to be climbed and to reach vibrational thermal equilibrium. The translational temperature overshoot for complex molecules can be tens of thousands Kelvin for shock-wave intensities characteristic of the detonation of explosives although the molecular degrees of freedom remain relatively cool. At such temperatures, electronic excitations become possible within the phonon-overheated zone due to electron-phonon coupling enhanced by the shock. Thus, it is reasonable to assume that for secondary explosives or any system suffering a rapid input of mechanical energy, initial processes occurring within the shock wave front must be electronic in origin as it is for most primary explosives, while those decomposing behind the front can arise from vibrational instabilities.

Here we concentrate on a microscopic electronic mechanism of shock-induced molecular decomposition. A number of authors have proposed that electronic excitations are involved in chemical processes involved with detonation, especially in the initial step. This has been reviewed by Faust, ${ }^{4}$ and recently addressed for secondary explosives. ${ }^{5,6}$ The essence of the idea is that a shock wave can generate molecules in highly excited electronic states, a mechanoelectronic effect. Thus the initiating reaction due to shock is taken to be an electronic mechanism.

In a series of papers, Gilman ${ }^{7}$ has emphasized the role of mechanical energy in solid-state reactions and specifically of HOMO-LUMO (highest occupied molecular orbital-lowest unoccupied molecular orbital) gap closure in molecules suffering shear strain. Further elaboration on the excitonic mechanism of detonation initiation for RDX (hexahydro1,3,5-trinitro-1,3,5-triazine) crystal is found in a recent informative study by Kunz and co-workers. ${ }^{6}$ The model, based on numerical results, suggests that the pressure inside the impact wave front reduces the band gap between valence and conducting bands and promotes the HOMO-LUMO transition within a molecule. ${ }^{6}$ Gilman's strain-induced HOMOLUMO gap closing, ${ }^{7}$ the Kunz group calculations, ${ }^{6}$ and our previous model $^{8}$ for solid state reactions have stimulated us to investigate in detail an electronic instability mechanism for molecular decomposition and its relation to the mechanoelectronic effect.

The calculational experiments by Kunz and co-workers ${ }^{6}$ have shown lattice deformations can significantly decrease the HOMO-LUMO gap for molecules and further demonstrate that any excess strain, such as that associated with a shock wave or defects, can further reduce the gap, as originally suggested by Gilman. ${ }^{7}$ For the specific case of RDX, the calculations clearly indicate that the gap closure leads a single $\mathrm{N}-\mathrm{NO}_{2}$ bond to break.

A detailed study of the photodecomposition ${ }^{5}$ of free RDX has demonstrated conclusively that its photodissociation around $5.50 \mathrm{eV}$ initially produces NO. This does not, however, define the reaction pathway producing the NO. Nevertheless, the study establishes that the $\mathrm{N}-\mathrm{NO}_{2}$ group is involved in the photodissociation of RDX as it appears to be in detonation.

As in all materials research, we seek structure-function relationships. In the case of energetic materials (henceforth taken as representative of all materials undergoing rapid input of mechanical energy), we look for the microscopic origin of the molecular multistability, a ground state with multiple minima, that is the essential property of such systems. The multistability is a manifestation of strong vibrational mixing between the ground and excited states. Thus, for energetic molecules we expect an important contribution of electron-molecular vibration coupling to the ground state properties. This naturally suggests an electronic mechanism for the first step of shock-induced reaction. Our hypothesis is that energetic materials' sensitivity is associated with those whose electronic structure changes under compression so electronic excitation of constituent molecules is favored. This electronic mechanism for nonequilibrium decomposition of a molecule within a shock compression is closely related to a change in reactivity index under stress, the molecular hardness. Gilman has examined the connection of mechanical stress and molecular hardness. ${ }^{9}$

Because solids are the primary concern for the shock initiation of reaction, our general theory of solid-state reactions ${ }^{8}$ can be applied. Indeed, in that prior work, a brief discussion of the shock initiation of reaction relating to the detonation problem was presented. Here we incorporate the above considerations into our general theoretical formulation for solid-state reactivity so that a specific model for the shock initiation of a chemical reaction and, as shall be seen, mechanochemical transformations in general, can be developed.

In this paper we next discuss the electron-driven instabilities due to stresses related to molecular hardness. Density functional theory (DFT) is then used to calculate the relevant properties for the RDX molecule. These results are subsequently related to the mechanoelectronic effect that is connected to local instability arising from gap closure. Finally, local mechanical instability and the electron-electron coupling relation to cooperativity is considered. 


\section{ELECTRON-DRIVEN INSTABILITIES}

The stress-induced chemical decomposition process within the compressed region due to shock, which must have an electronic mechanism, is similar to photoinduced structural changes. ${ }^{10}$ In both cases, a molecule undergoes perturbation for such a short period that there is insufficient time to establish thermal equilibrium in the system, and, as in photoexcitation, the energy goes directly into electronic degrees of freedom. Mechanical shock, as with optical excitation of molecules, creates a sudden change in the electron distribution, thereby destroying the balance of electron-mediated interatomic forces that dictate the electronic ground state. There is an experimental observation that substantiates this view. Shock decomposition products of RDX molecules differ from those found from thermal decomposition and are identified with those of photochemical decomposition. ${ }^{11,12}$ This suggests decomposition within the shock front occurs by an electronic process since it is known that photochemical decomposition proceeds through electronic excitation. ${ }^{5}$

For shock-induced changes, one may ask what are the roles of molecular electronic instability and cooperative interactions in the solid. For a strongly coupled electron-lattice system, such as with energetic materials, the stability of a cluster of $m$ perturbed molecules can be considered for illustrative purposes. The crystal is treated as an elastic medium described by the mechanical susceptibility, $\mathbf{X}(\mathbf{q})$. The inverse of this matrix is the dynamical matrix, with eigenvalues, $\omega^{2}\left(\mathbf{q}_{j}\right)$, and eigenvectors, $\mathbf{e}\left(\mathbf{q}_{j}\right)$, that characterize phonons of both intermolecular and intramolecular origin in the $j$ th dispersion branch for a given wave vector, q. When a molecule at site $n^{\prime}$ is perturbed by the mechanical energy, $E$, it and the surrounding lattice deform site $n$, by the amount,

$$
\langle\mathbf{Q}(n)\rangle=\sum_{n} \mathbf{X}\left(n, n^{\prime}\right) \mathbf{F}\left(n^{\prime}\right),
$$

expressed in terms of the coordinate vector, $\mathbf{Q}$. This vector is composed of external (translational and rotational) as well as intramolecular degrees of freedom. The cause of the structural change is the force, $\mathbf{F}\left(n^{\prime}\right)=-\left[\partial E / \partial \mathbf{Q}\left(n^{\prime}\right)\right]_{0}$, generated at the perturbed site due to coupling between the electronic structure of the molecule and the nuclear structure of the molecules and the lattice. The force is a measure of chemical pressure and, as an analog of local stress, can be described in terms of elastic multipoles. ${ }^{8}$ The response function, $\mathbf{X}\left(n, n^{\prime}\right)$, is the inverse Fourier transform of $\mathbf{X}(\mathbf{q})$.

Here, a comment about the notation and its relation to elasticity theory is required. Eq. (1) is analogous to the stress-strain relationship for a continuous medium. For a particular molecule in a crystal, instead of a deformation tensor, we use the vector, $\mathbf{Q}(n)$, that describes molecular distortions in terms of traditional external and internal coordinates. Similarly, the perturbation is expressed as a force vector $\mathbf{F}\left(n^{\prime}\right)$, and consequently Eq. (1) is vectorial and not tensorial, as it would be for a continuous medium. Consequently, throughout the paper the displacement vector, $\mathbf{Q}(n)$, will be used to express a molecular deformation.

Although one could represent the mechanical properties of a molecule by a molecular elastic tensor, it would invite comparison with the elastic tensor associated with bulk material and contravene established conventions in molecular physics. The concepts of isotropic and shear strain are not particularly useful at the molecular level and have not, to our knowledge, been employed in a formal construction. Force constants and normal coordinates are normative in discussions of molecular distortion and strain and we conform to those conventions. This is consistent with the fact that the elastic constants that appear in an elastic tensor are themselves functions of the force constants of the bodies they describe. In this sense, a molecular elastic tensor would be a derived quantity of doubtful practical value at the microscopic level. In a later discussion (Sec. III) of molecular interactions with mechanical fields, we show the utility of elastic multipoles that may be easily represented as tensors.

Denoting molecules perturbed by compression by the operator $\sigma(n)=1$ and unperturbed ones by $\sigma(n)=0$, we have previously shown that the energy change due to $m$ perturbed molecules is $\Delta E=m E_{0}+\Phi_{\text {def }}$, where the deformation energy is ${ }^{8}$

$$
\begin{aligned}
\Phi_{\mathrm{def}} & =-\frac{1}{2} \sum_{n} \sum_{n^{\prime}} \mathbf{F}^{T}(n) \mathbf{X}\left(n, n^{\prime}\right) \mathbf{F}\left(n^{\prime}\right) \sigma(n) \sigma\left(n^{\prime}\right) \\
& =-\frac{1}{2} \sum_{n} \sum_{n^{\prime}} J\left(n, n^{\prime}\right) \sigma(n) \sigma\left(n^{\prime}\right) .
\end{aligned}
$$

The $n=n^{\prime}$ term corresponds to the self-deformation energy, $\Phi_{\text {self }}=-(1 / 2) J(n, n)$, and renormalizes the on-site energy, $E_{0}$. It is the solid-state analog of the solvent reorganization energy. The energy change of the system is therefore,

$$
\Delta E=m \delta-\frac{1}{2} \sum_{n} \sum_{n^{\prime}} J\left(n, n^{\prime}\right) \sigma(n) \sigma\left(n^{\prime}\right),
$$

where $\delta=E_{0}-1 / 2 J(n, n)$ is the effective energy needed to perturb a molecule in a crystal. It describes an energetic effect due to both molecular volume and shape changes with respect to the unperturbed state. Equation (3) expresses the balance between the expense of energy to perturb the molecules (first term) and the gain of energy due to cooperative, phonon-mediated interactions. In case of an electronic excitation of energy $E_{0}$, the self-deformation energy may be viewed as the energy gain in a process of structural intramolecular and lattice relaxations and the second term represents an additional gain arising from cooperative relaxation of the $m$ excited molecules. The athermal system becomes unstable toward a conformational change when $\Delta E \leqslant 0$. A change of bonding cannot be predicted, but it is reasonable that this is also the condition for the most probable initial step in spontaneous decomposition. The reaction can occur locally or cooperatively.

The condition for a local instability is $\delta=0$, which expresses the compensation of electronic, $E_{0}$, and mechanical, $\Phi_{\text {self }}$, energies. This can be understood two ways depending upon which energy has been supplied to the system by a direct action. For example, supply of electronic energy by photoexcitation allows an excited site to relax to a deformed site. A molecule in a crystal will therefore deform, as will its nearest surroundings. In this case, the energy is transferred 
from electronic to vibrational degrees of freedom, i.e. by electronic relaxation. This transfer occurs by coupling between these degrees of freedom and is quantified by the force, $\mathbf{F}(n)$ defined above. It is the same coupling that is involved in the reverse process of transferring mechanical energy from vibrational degrees of freedom to electronic ones. Such a process may occur upon introduction of a localized deformation energy comparable to the gap $\left(E_{0}\right)$ in the electronic energy levels of a molecule. The energy gap corresponds to the energy difference between the HOMO and LUMO orbitals. The instability condition, $E_{0}=\Phi_{\text {self }}$, defines the degree of deformation required to promote an electron from the HOMO to the LUMO. In a crystal, of course, the HOMO and LUMO form the highest occupied crystal band, the valence band, and the lowest unoccupied crystal band, the conduction band, respectively. However, energetic materials are insulators and their bands are very narrow. Thus, in a first approximation focus may be on a single site. The criterion for the local instability then becomes $E_{0}=I$ $-A=\Phi_{\text {self }}$, where the HOMO-LUMO gap has been described by the difference between the ionization energy $(I)$ and the electron affinity $(A)$.

\section{LOCAL ELECTRONIC INSTABILITY AND MOLECULAR HARDNESS}

Modern reactivity is described within density functional theory (DFT) that introduces hardness $(\eta)$ and softness $(\sigma$ $=1 / \eta)$ as $^{13}$

$$
\eta=\frac{1}{2}\left(\frac{\partial \mu}{\partial N}\right)_{\nu(\mathbf{r})},
$$

where $\nu(\mathbf{r})$ is an external potential, $N$ stands for the number of electrons and $\mu$ is the chemical potential,

$$
\mu=\left(\frac{\delta E[\rho(\mathbf{r})]}{\delta \rho(\mathbf{r})}\right)_{\nu(\mathbf{r})}=\left(\frac{\partial E}{\partial N}\right)_{\nu(\mathbf{r})},
$$

where $\rho(\mathbf{r})$ is the electron density and $E[\rho(\mathbf{r})]$ is the energy functional. ${ }^{14,15}$ It follows that hardness is the second derivative of energy with respect of the number of electrons, ${ }^{15}$

$$
\eta=\frac{1}{2}\left(\frac{\partial^{2} E(N, \nu(\mathbf{r}))}{\partial N^{2}}\right)_{\nu(\mathbf{r})} .
$$

Taking the finite difference approximation for the curvature of $E[N, \nu(\mathbf{r})]$, one obtains the following formulas for hardness, $\eta$, and chemical potential, $\mu$, respectively, ${ }^{13}$

$$
\eta=(I-A) / 2 \text { and } \mu=(I+A) / 2,
$$

where $I$ is the ionization potential and $A$ is the electron affinity of a molecule at equilibrium. This is a working definition of hardness where the HOMO-LUMO gap is approximated within Koopman's theorem by the energy difference, $I-A$. A small gap increases quantum mixing and therefore enhances chemical reactivity. Thus, the softness is the measure of reactivity of a molecule - the smaller the gap, the more readily a unimolecular reaction can occur.

The hardness, a molecular quantity, provides a convenient way to handle the energetics of the HOMO-LUMO gap. Although a molecular quantity, it may be used in the analysis of the role of mechanical energy upon molecular energetics in much the same way that the free molecule energy levels are used in the exciton theory of solids. A more exact treatment would actually deal with a conduction bandvalence band gap but, because in molecular crystals these bands are very narrow, the HOMO-LUMO gap retains close connection to molecular properties. This is of particular relevance in the design of energetic materials. Thus, while a formulation using band theory would be more exact, it probably would offer little additional insight regarding this initial step.

The energy gap also has its effect on molecular polarizability. A small energy gap means that a manifold of excited states lies near the ground state; a small gap means high polarizability. Therefore, electronically (chemically) soft and highly polarizable molecules are the most sensitive to unimolecular decomposition. Indeed, it has been shown ${ }^{16}$ that there is a reasonable correlation between the "drop height sensitivity" of 50 organic explosives and the HOMOLUMO energy gap. We shall analyze this correlation from a molecular point of view and examine how hardness depends on molecular deformation.

In the simplest approximation, one can express the hardness of a deformed molecule as

$$
\eta=\eta_{0}+\sum_{i}\left(\frac{\partial \eta}{\partial \mathbf{Q}_{i}}\right)_{N} \cdot \mathbf{Q}_{i}
$$

where $\eta_{0}$ is the hardness of a nondeformed molecule at equilibrium. Molecular deformation is expressed in terms of an atomic displacement set, $\left\{\mathbf{Q}_{i}\right\}$. Following the derivation presented earlier, ${ }^{17}$ one finds that the hardness' dependence on the $i$ th atomic displacement is given as

$$
\mathbf{G}_{i}=\left(\frac{\partial \eta}{\partial \mathbf{Q}_{i}}\right)_{N} \cong-\frac{1}{2}\left(\mathbf{F}_{i}^{+}+\mathbf{F}_{i}^{-}\right),
$$

where

$$
\mathbf{F}_{i}=-\left(\frac{\partial E(N, \nu(\mathbf{r}))}{\partial \mathbf{Q}_{i}}\right)_{N}
$$

is the Hellman-Feynman force. ${ }^{18} \mathbf{F}_{i}^{+}$and $\mathbf{F}_{i}^{-}$are the total forces acting on the $i$ th nucleus in the positively and the negatively charged molecules, respectively. $\mathbf{G}_{i}$ has been called the nuclear stiffness ${ }^{17}$ or nuclear Fukui function ${ }^{19}$ in contrast to the nuclear reactivity as defined and discussed by Cohen et $_{\text {al. }}{ }^{20}$

Similarly, one obtains an expression for the deformation dependence of the chemical potential

$$
\mu=\mu_{0}+\sum_{i}\left(\frac{\partial \mu}{\partial \mathbf{Q}_{i}}\right)_{N} \cdot \mathbf{Q}_{i},
$$

where

$$
\boldsymbol{\varphi}_{i}=-\left(\frac{\partial \mu}{\partial \mathbf{Q}_{i}}\right)_{N}=\left(\frac{\partial \mathbf{F}_{i}}{\partial N}\right)_{\nu(\mathbf{r})}=\frac{1}{2}\left(\mathbf{F}_{i}^{-}-\mathbf{F}_{i}^{+}\right)
$$

has been introduced as the nuclear reactivity index. ${ }^{17,19,20}$

The forces $\mathbf{G}_{i}$ and $\mathbf{F}_{i}$, acting on the $i$ th atom at the distance $\mathbf{r}_{i}$ from the center of gravity of a molecule form a 
distribution of forces. Thus, the nuclear reactivity can be alternatively expressed in terms of elastic multipoles ${ }^{8}$ and, in particular, the elastic dipole tensor with components $P \mu \nu$ $=\sum_{i} F_{i}^{\mu} Q_{i}^{\nu}+F_{i}^{\nu} Q_{i}^{\mu}$ represents a molecular strain/stress tensor, i.e., essenitially serves as a molecular elastic tensor, where off-diagonal terms represent shear components. However, this formulation is more general and easier to work with in terms of the forces associated with molecules than a true molecular elastic tensor would be. Nevertheless, such a representation suggests that the nuclear reactivity can be expressed as a tensor, just as, for example, $d$ orbitals may be represented as tensors, and thus, the usefulness of tensor properties can be retained.

It is more convenient to work in the space of molecular normal mode coordinates, $Q_{\alpha}$, and transform indices into that space,

$$
\begin{aligned}
G_{\alpha}=\left(\frac{\partial \eta}{\partial Q_{\alpha}}\right)_{N} & =\sum_{i}\left(\frac{\partial \eta}{\partial \mathbf{Q}_{i}}\right)_{N} \cdot\left(\frac{\partial \mathbf{Q}_{i}}{\partial Q_{\alpha}}\right)_{N} \\
& =\sum_{i} \mathbf{G}_{i} \cdot\left(\frac{\partial \mathbf{Q}_{i}}{\partial Q_{\alpha}}\right)_{N} .
\end{aligned}
$$

$G_{\alpha}$ indicates how hardness decreases due to a molecular deformation that is described by the atomic displacements of the $\alpha$ th normal mode coordinate. For the chemical potential derivative we obtain

$$
\begin{aligned}
\varphi^{\alpha}=-\left(\frac{\partial \mu}{\partial Q_{\alpha}}\right)_{N} & =-\sum_{i}\left(\frac{\partial \mu}{\partial \mathbf{Q}_{i}}\right)_{N} \cdot\left(\frac{\partial \mathbf{Q}_{i}}{\partial Q_{\alpha}}\right)_{N} \\
& =\sum_{i} \varphi_{i} \cdot\left(\frac{\partial \mathbf{Q}_{i}}{\partial Q_{\alpha}}\right)_{N} .
\end{aligned}
$$

Consider a molecule in a stress-free state. Its chemical potential is $\mu_{0}=(I+A) / 2$ and its hardness is $\eta_{0}=(I-A) / 2$. The change in the energy of the molecule due to its deformation and the related change in the electron density, strictly speaking, the change in the number of electrons in the frontier orbitals, $\Delta N$, is

$$
\Delta E=\mu(\Delta N)+\eta(\Delta N)^{2}+\frac{1}{2} \sum_{\alpha} k_{\alpha} Q_{\alpha}^{2},
$$

where the chemical potential and hardness are approximated by their linear dependencies on the normal mode displacement amplitudes, $Q_{\alpha}$, and $k_{\alpha}$ is the force constant related to the $\alpha$ th normal mode frequency, $\omega_{\alpha}$. Due to the change in number of electrons, $\Delta N$, there is molecular deformation along the normal mode coordinate

$$
\left\langle Q_{\alpha}\right\rangle=\frac{\varphi_{\alpha}(\Delta N)-G_{\alpha}(\Delta N)^{2}}{k_{\alpha}}
$$

when $\Delta E$ is at a minimum with respect to $Q_{\alpha}$

$$
\left(\frac{\partial \Delta E}{\partial Q_{\alpha}}\right)_{N}=0
$$

and the electronic part of the energy is renormalized,

$$
\begin{aligned}
\Delta E= & \mu_{0}(\Delta N)+\eta_{0}(\Delta N)^{2} \\
& -\frac{1}{2} \sum_{\alpha} \frac{\left(\varphi_{\alpha}(\Delta N)-G_{\alpha}(\Delta N)^{2}\right)^{2}}{k_{\alpha}} .
\end{aligned}
$$

The renormalized hardness of a molecule is

$$
\eta=\eta_{0}-\frac{1}{2} \sum_{\alpha} \frac{\varphi_{\alpha}^{2}}{k_{\alpha}}
$$

where modes that represent the molecular deformation will contribute most to decreasing the molecular hardness.

Even if the contribution of a single mode is not significant, the number of normal modes that can contribute to the renormalization is important for polyatomic molecules. The term that renormalizes the hardness is the molecular deformation energy that is analogous to the small polaron binding energy. ${ }^{8}$ It is the energy that a molecule gains in a process of intramolecular relaxation when an electron is promoted from the HOMO to the LUMO. Thus, the condition $\eta \rightarrow 0$ signifies promotion of an electron from the HOMO to the LUMO without any energy cost. This corresponds to an unstable state of a molecule with strong electron-molecular vibrational coupling.

Which normal mode coordinate distortions contribute to the change in the HOMO-LUMO energy gap? What are the selection rules imposed on the coupling constants? These questions have been discussed by Lipari et al. ${ }^{21}$ whose symmetry arguments show that only totally symmetric molecular vibrational modes couple linearly to nondegenerate molecular orbitals. If ionized states of a molecule are nondegenerate, the forces, $\left(\partial \eta / \partial \mathbf{Q}_{i}\right)_{N}$ and $\left(\partial \eta / \partial Q_{\alpha}\right)_{N}$, possess molecular symmetry. However, the normal coordinate displacements, $\left(\partial \mathbf{Q}_{\mathrm{i}} / \partial Q_{\alpha}\right)_{N}$, are not necessarily totally symmetric. The criterion for nonzero coupling, as defined by Eq. (13), is that the irreducible representation of a molecular orbital, $\Gamma_{\beta}$, be contained in the direct product of that representation and the representation of the $\alpha$ th molecular vibration, $\Gamma_{\alpha}, \Gamma_{\beta} \otimes \Gamma_{\alpha} \in \Gamma_{\beta}$. Thus, the set of normal mode coordinate deformations ( $Q$ deformations) that contributes to the linear coupling constants contains only the totally symmetric mode distortions for low-symmetry molecules. For a molecule at a crystal site, this requires totally symmetric $Q$-distortions under the site symmetry. In the case of shock-induced reaction (SIR), the shock wave, in particular the associated shear stress, destroys the crystal and site symmetries and effectively allows linear coupling with all molecular normal mode displacements.

In analyzing an isolated molecule, particularly an often high-symmetry secondary explosive, the role of degeneracy must be considered. In this case, the forces do not possess molecular symmetry and nonzero coupling constants are obtained for those $Q$ distortions of the same symmetry as the forces. For example, when the HOMO is doubly degenerate ( $E$ symmetry) and an electron is removed from the molecule, the forces that drive the molecule to a new global minimum break the molecular symmetry according to the Jahn-Teller theorem. This means that for high symmetry molecules, the molecular electronic instability determined by the condition, $\eta \rightarrow 0$, may result in nontotally symmetric decomposition of a 
molecule. This effect is clearly illustrated by the following numerical calculations performed for the RDX molecule.

\section{CALCULATIONS FOR THE RDX MOLECULE}

RDX is an important secondary explosive that for the last few years has served as a model system for study of the details of detonation and molecular decomposition. For this reason it has been chosen to illustrate our model. The main goal is to understand and predict mechanistic details of the initial step in the shock-induced molecular decomposition mechanism. Many experimental studies have been directed toward this problem and various reaction pathways have been proposed (for the list of references, see Ref. 22). In short, there is experimental evidence for two pathways: (i) concerted ring fission to three $\mathrm{CH}_{2} \mathrm{~N}_{2} \mathrm{O}_{2}$ radicals, and (ii) nonsymmetric $\mathrm{N}-\mathrm{NO}_{2}$ bond rupture. Recent UV photolysis experiments ${ }^{23-27}$ support pathway (ii), infrared multiphonon dissociation experiments ${ }^{28}$ conclude that pathway (i) is the dominant channel, and very recent experiments on photodissociation of RDX suggest that $\mathrm{NO}$ is an initial product of decomposition. ${ }^{5}$

The mechanism of unimolecular decomposition of RDX has been studied numerically via $a b$ initio quantum chemical methods (Ref. 22 and references therein). These studies have provided support for both pathways with some favor for homolytic $\mathrm{N}-\mathrm{N}$ bond fission. In particular, after carrying out extensive high-level DFT calculations, Wu and Fried $^{29}$ have concluded that $\mathrm{N}-\mathrm{NO}_{2}$ bond rupture is the dominant channel for the decomposition of RDX. Very recent DFT studies ${ }^{22}$ have suggested a third pathway: successive HONO radical elimination as the most exothermic primary RDX decomposition channel.

These results may be compared to the predictions of our renormalized hardness model. There is, of course, a fundamental difference between these approaches. In the case of the $a b$ initio studies, the energy barriers are calculated for assumed pathways, e.g., energy changes along molecular coordinates which are involved in the pathway. In this model of renormalized hardness, all coordinates allowed by symmetry considerations contribute to the renormalization. This permits the pathway for molecular decomposition to be estimated using those coordinates with the largest contribution to the molecular deformation energy.

The calculations of structure, normal mode frequencies and forces for RDX molecule were performed using the DFT method $^{15}$ in conjunction with the Becke ${ }^{30}$ (exchange) and Lee-Young-Parr ${ }^{31}$ (correlation) functionals. The geometry optimization employed a quasi Newton-Raphson procedure ${ }^{29}$ and the basis set $6-31 \mathrm{G}^{* *}$ has been used. The DFT calculations have been performed with Gaussian 94 code. ${ }^{32}$ Geometry optimization was obtained within $C_{3 v}$ symmetry constraints. The resulting optimized geometry is presented in Fig. 1 with the values of the bond angles and lengths listed in Table I of the supporting information. ${ }^{33}$ The results are in very good agreement with the $a b$ initio studies of Rice and Chabalowski ${ }^{34}$ and the experimental data they cite. Especially, bond lengths are within $1 \%$ of experimental results. Bond angles are not reproduced as well, but they agree with the theoretical results of Ref. 34. Atomic

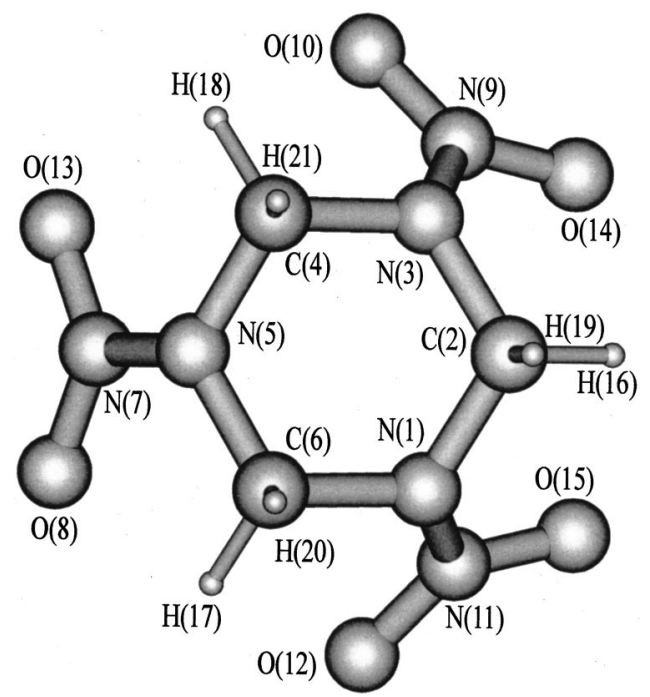

FIG. 1. Ball and stick drawing of the RDX molecule according to the calculated optimized geometry.

Hellman-Feynman forces were calculated for a positively and negatively charged RDX molecule as defined by Eq. (10) with values available in Table II of the supporting information. ${ }^{33}$ These forces have been used to calculate the nuclear reactivity indices, Eqs. (9) and (12), and are assigned to every atom in Table III of the supporting information. ${ }^{33}$ The combination of forces corresponding to the nuclear reactivity indices is shown in Fig. 2. The most significant force acts along one $\mathrm{N}-\mathrm{NO}_{2}$ bond as a consequence of the JahnTeller effect and indicates that this bond is most affected by the stress-induced electronic mechanism of decomposition.

Normal mode frequencies, their symmetries, and the reactivity indices projected into normal coordinate space are available in Table IV of the supporting information. ${ }^{33}$ The frequencies are in agreement with experimental data. ${ }^{35}$ These results show that doubly degenerate $Q$-distortions contribute significantly to renormalization of the hardness. The RDX molecule has a twofold degenerate HOMO ( $E$ symmetry)

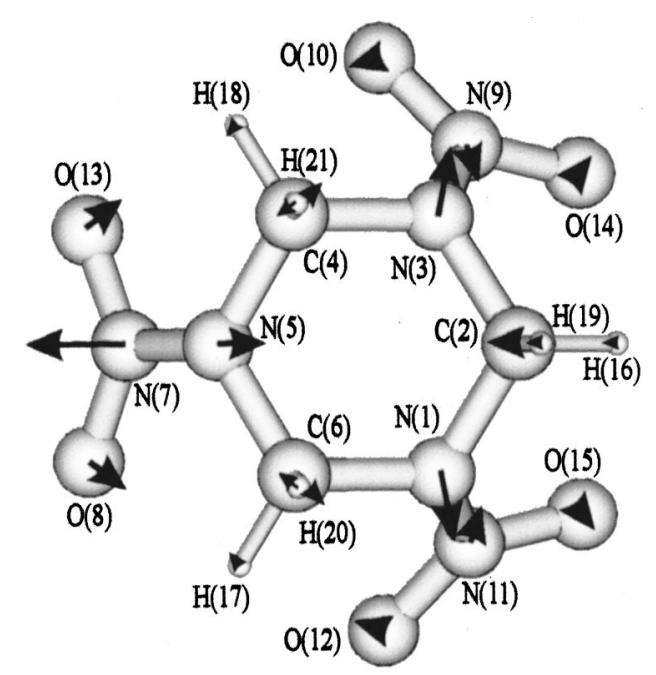

FIG. 2. RDX nuclear reactivity indices $\left(\varphi_{i}\right)$ for top view of the molecule. Magnitudes are proportional to line lengths at each nucleus, $i$. 


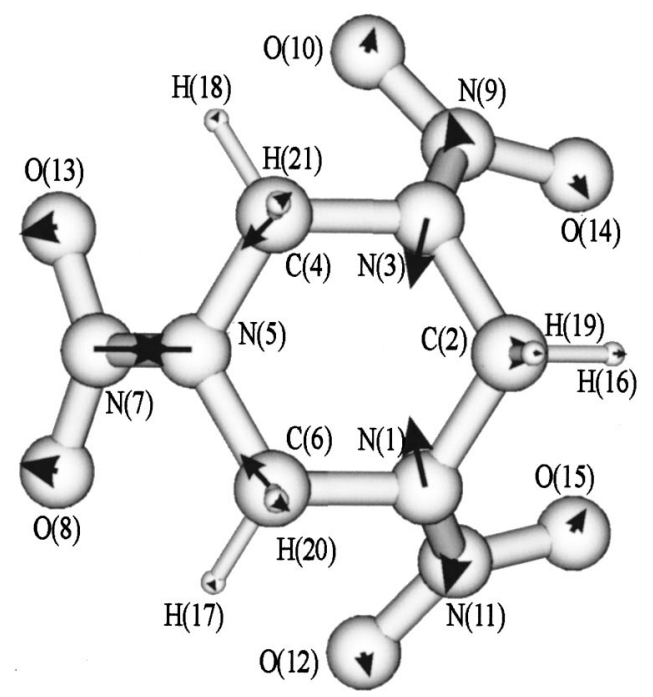

FIG. 3. RDX nuclear stiffness $\left(\mathbf{G}_{i}\right)$ for top view of the molecule. Magnitudes are proportional to line lengths at each nucleus, $i$.

with energy $E_{\mathrm{HOMO}}=-8.32 \mathrm{eV}$. Therefore, by removing an electron from RDX, the electronic state of the ion becomes doubly degenerate and, according to the Jahn-Teller theorem, the resulting forces lower the molecular symmetry to $C_{s}$. This is shown in Fig. 3 where RDX nuclear stiffnesses are represented; numerical values are collected in Table IV of the supporting information. ${ }^{33}$

The global hardness, $\eta_{0}$, of the RDX molecule is calculated to be $5.16 \mathrm{eV}$. The molecular deformation energy corresponding to promotion of an electron from the HOMO to the LUMO renormalizes the hardness to $7.99 \mathrm{eV}$ (see Table IV of supporting information). ${ }^{33}$ From these calculations it follows that the largest influence decreasing the hardness is due to (1) extending the $\mathrm{N}-\mathrm{N}$ bond and (2) forcing the carbon atom opposite the $\mathrm{N}-\mathrm{N}$ bond from the ring. These deformations correspond to the suggested pathways (ii) and (i), respectively. However, the $\mathrm{N}-\mathrm{N}$ bond extension has a greater effect on the hardness than ring fission. Moreover, the nuclear reactivity index shows that the most significant contribution to the decrease of hardness comes from the nitrogen and oxygen atoms of the nitro group. In other words, changes in position of these atoms lead to the largest change in the reactivity index of the molecule. This indicates that the most probable, distortion-induced, initial, unimolecular reaction in RDX is breaking of an $\mathrm{N}-\mathrm{NO}_{2}$ bond. These results should be considered to be general and demonstrate how a possible shock-induced reaction pathway can be constructed from a linear combination of $Q$ deformations. For this, it is important to identify those $Q$ distortions that contribute most to the hardness renormalization. For RDX these are all characterized by an $\mathrm{N}-\mathrm{N}$ bond extension that is in phase with the ring deformation and are listed in Table $\mathrm{V}$ of the supporting information. ${ }^{33}$

The flexibility of RDX that is reflected by its low frequency intramolecular vibrations is a key factor in the determination of the pathway for SIR. This indicates that the low energy intramolecular vibrations may also be considered as "doorway modes" for the electronic mechanism of decom- position as well as for the vibrational up-pumping mechanism. ${ }^{3}$ This also explains why the empirical design of energetic materials has resulted in systems that are quite conformationally labile.

Although quantum chemistry calculations are important to describe complex systems, understanding such systems with desired predictive power and analysis requires insights obtained from conceptually manageable models. The ensuing sections are devoted to such a description.

\section{THE MECHANOELECTRONIC EFFECT}

The molecular distortion that accompanies the promotion of an electron from the HOMO to the LUMO is found from the condition for a minimum of the total energy of a molecule. Thus, the hardness is expressed alternatively as

$$
\eta=\eta_{0}-\frac{1}{2} \sum_{\alpha} k_{\alpha}\left\langle Q_{\alpha}\right\rangle^{2}
$$

This equation is fundamental to understanding the increase in reactivity of a molecule due to a molecular deformation.

When an externally applied force deforms a molecule, the mechanical energy introduced into the molecule is transferred into the electronic degrees of freedom. Therefore, the instability condition, $\eta \rightarrow 0$, determines the critical distortion, $\Sigma_{\alpha} k_{\alpha}\left\langle Q_{\alpha}\right\rangle_{c}^{2}=(I-A)$, needed to promote an electron from the HOMO to the LUMO via the mechanoelectronic effect. We refer to such a state as a distortion-induced molecular electronic (HOMO/LUMO) degeneracy (DIMED) that is an analog to the Jahn-Teller effect that may be viewed as an "inverse" Jahn-Teller effect, since it appears with structurally relaxed electronic states. One can speculate about such a state for a molecule with a negative hardness created by molecular deformation. It could be thought of as a molecule with negative polarizability, a molecular state with anomalous polarization arising as a product of bond distortions produced by a self-trapping effect such as in photoexcitation. ${ }^{36}$

It is instructive to set the above considerations into the perspective of potential energy surfaces. A molecule in a crystal is described by an energy surface in a multidimensional space with a critical path along which the potential decomposition reaction travels. From the above calculations for RDX, this path is a combination of those normal mode coordinates that contribute the most to the hardness renormalization. Assume the pathway to be described by coordinate $Q$, which describes an effective $\mathrm{N}-\mathrm{N}$ stretching mode with force constant, $k$, for the RDX molecule. The ground state of the molecule is represented by the parabolic potential, $E_{R}=(1 / 2) k Q^{2}$. Now consider the same molecule, but with electronic structure changed due to promotion of an electron from the HOMO to the LUMO. For the same coordinate, we assume that the energy of the molecule depends linearly on the $Q$ deformation, and that molecular elasticity does not significantly change due to the promotion of the electron. The potential energy for the state with the promoted electron is represented by the parabolic potential, $E_{P}=(I$ $-A)+\Gamma Q+(1 / 2) k Q^{2}$ where $\Gamma$ is the force due to deformation and the other arguments have their usual meanings. 


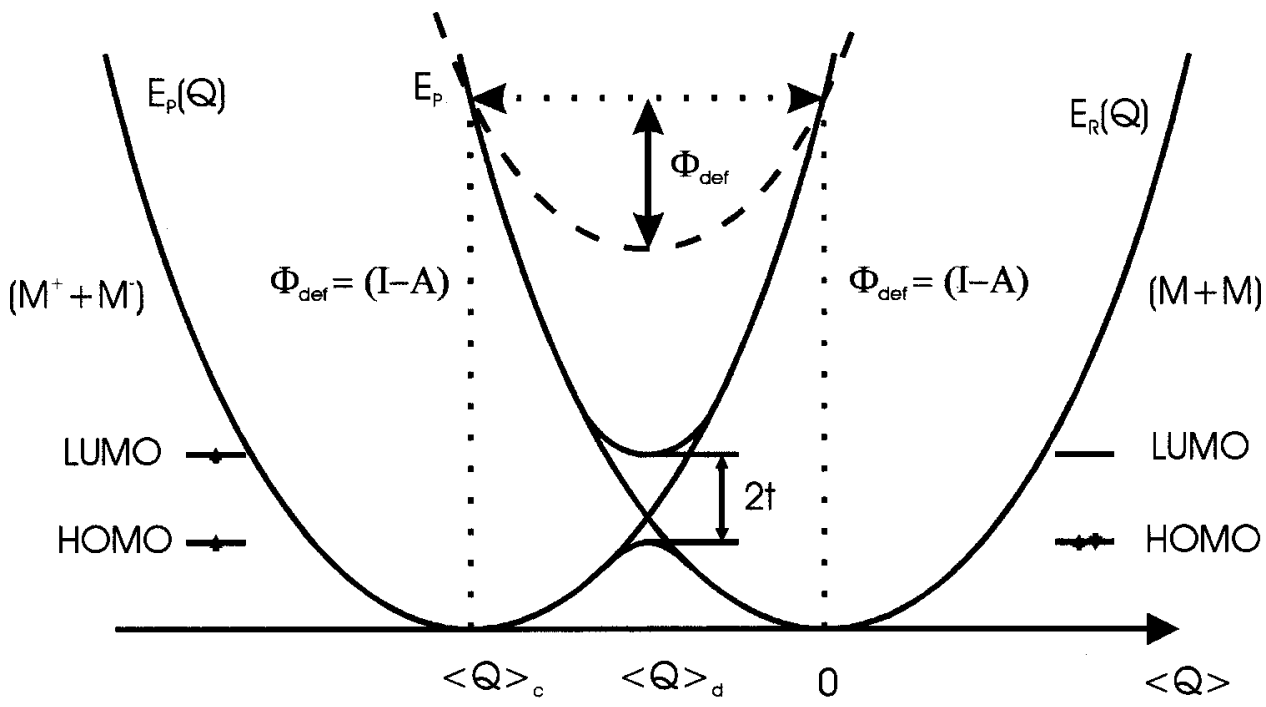

FIG. 4. Diabatic potentials, $E_{R}$ and $E_{P}$, illustrating deformation-induced molecular electronic degeneracy (DIMED). $Q$ is the vibrational normal coordinate, $\Phi_{\text {def }}$ is the deformation potential, and $2 t$ is the bandwidth of the deformation-induced state.
These two potentials, schematically shown in Fig. 4, are diabatic energy curves; they correspond to two different electronic structures of the molecule. The scheme in Fig. 4 applies equally well to an electron transfer reaction between two molecules (M), $\mathrm{M}+\mathrm{M} \Rightarrow \mathrm{M}^{+}+\mathrm{M}^{-}$. This means that, qualitatively, the process of single molecule excitation, promotion of an electron from the HOMO to the LUMO, and creation of charge-transfer excitation, which is the essence of the mechanoelectronic effect, can be discussed within the same framework.

What happens to a molecule when it becomes mechanically deformed? Deformation may be viewed as raising the molecule's energy from the bottom of the $E_{R}$ potential well to the value of the deformation energy, $\Phi_{\text {def }}=k\langle Q\rangle^{2}$. Since the electrons immediately follow the deformation, there is vertical relaxation to a new state-a deformed molecule with an electron in the HOMO and in the LUMO. If the bottom of the new state's potential well lies above the reactant's energy as shown by the dashed curve in Fig. 4, the new state is unstable and immediately relaxes back into the minimum of energy for the reactant state. Such a situation happens when the applied deformation, $\langle Q\rangle$, is insufficient to compensate for the electronic energy expense, $(I-A)>\Phi_{\mathrm{def}}$. However, when the molecule is driven to critical deformation, the new state of the molecule has equally occupied HOMO and LUMO and a deformation geometry, $\langle Q\rangle_{\mathrm{cd}}$, which is stable. Of course, the inequality, $(I-A)<\Phi_{\text {def }}$, will cause spontaneous creation of unstable molecules in the new electronic state via DIMED.

\section{MOLECULAR ELECTRONIC DEGENERACY ARISING FROM GAP CLOSURE}

The diabatic energy curves describe formation of a multistable ground state due to an applied mechanical field. This is an adiabatic state with multiple minima. The multistability of the ground state is an essential requirement for having molecules susceptible to mechanochemical reaction in a condensed phase. Recent extensive calculations have shown RDX has a variety of conformations that are close in energy $^{30}$ and thus consistent with this model. The diabatic energy functions have been approximated by parabolic potentials, but they would be more realistic if represented by Morse functions such as have been used in numerical simulations for model explosive molecules. ${ }^{37,38}$

Consider formation of an adiabatic ground state from quantum mixing of the diabatic states as in Fig. 4. The states are either those corresponding to a single molecule with either a LUMO empty $\left(E_{R}\right)$ or occupied $\left(E_{P}\right)$, or to a dimer without and with a transferred electron. We denote the quantum mixing by $t$, and assume the states are of proper symmetry for mixing. The adiabatic ground state energy is then

$$
E_{G}=\frac{1}{2}\left[E-\left(E^{2}+4 t^{2}\right)^{1 / 2}\right]+\frac{1}{2} k Q^{2},
$$

where, $E=(I-A)+\Gamma Q$. This can be generalized for many electron-molecular vibration couplings as considered above. The degree of transformation of a molecule from the ground state to one with a promoted (intramolecular or intermolecular) electron can be calculated as the derivative of the ground state energy with respect to the "field," the electronic energy, $E$. The result is

$$
\rho(Q)=\frac{1}{2}\left[1-E\left(E^{2}+4 t^{2}\right)^{-1 / 2}\right] .
$$

For sufficiently small mixing, the nondeformed molecule remains in its ground state. But when the electronic energy decreases $(I-A+\Gamma Q) \rightarrow 0, \rho(Q) \rightarrow 1 / 2$, and an energy level, which has $2 t$ bandwidth and population $\frac{1}{2}$, is formed. Thus, we have obtained the condition for the critical deformation, $\langle Q\rangle_{\mathrm{cd}}=-(I-A) / \Gamma$, which causes HOMO-LUMO gap closure-the state which Gilman ${ }^{6}$ has called local metallization and which is a special case of DIMED.

Figure 4 gives a visual interpretation of the critical deformation energy. It corresponds to the maximum in the ground state energy encountered on passing from the normal state to a state with a promoted electron, or in case of a dimer, to a state with degree of charge transfer of $\frac{1}{2}$. We conclude that the deformation required to cause degeneracy of the nonrelaxed HOMO/LUMO states is smaller than, and differs from, the critical deformation required to promote an electron from the HOMO to the LUMO. 
The gap closure and mechanoelectronic mechanisms are complementary. In order to cause a HOMO-LUMO gap closure for a molecule, it is required that it be deformed by the amount $\langle Q\rangle_{\mathrm{cd}}$. This causes formation of an electronic level of width $2 t$ and population $\frac{1}{2}$. In order to stabilize that state, it is sufficient to deform the molecule to the critical deformation geometry, $\langle Q\rangle_{\mathrm{cd}}$. This means that a molecule with an electron promoted from the HOMO to the LUMO has been created. However some, but less than sufficient, deformation of a molecule causes a nonrelaxed HOMO-LUMO degeneracy. Without the extra energy required to cross the $\langle\Phi\rangle_{\text {crit }}$ barrier, the molecule may relax to its unstressed ground state (lower energy) minimum. The extra energy that stabilizes the process is supplied by cooperative interactions.

\section{LOCAL MECHANICAL INSTABILITY}

We now analyze an important consequence for molecular mechanics that follows from the local electronic instability. Within the simplified model we locate the frequency, $\Omega$, of a critical mode under deformation. The square of the frequency is given by the second derivative of the ground state energy with respect to the mode coordinate, $Q$. The result is

$$
\Omega^{2}=\omega^{2}-\gamma^{2} \chi_{\mathrm{el}},
$$

where

$$
\chi_{\mathrm{el}}=\frac{2 \rho(1-\rho)}{\left[E^{2}+4 t^{2}\right]^{1 / 2}}
$$

is the electronic susceptibility, $\omega^{2}$ is the force constant, $k$, divided by a reduced mass, and $\gamma$ is the Grüneisen parameter. The denominator is the effective energy gap, and the molecular deformation that causes the gap closure drives the susceptibility towards infinity, i.e., causes the electronic instability. However, the immediate effect of the molecular deformation in closing the gap is to decrease the critical mode frequency. The molecule becomes mechanically softer and the limit of its mechanical stability is $\Omega^{2} \rightarrow 0$. This is, of course, molecular decomposition according to the atomic displacements defined by the critical mode coordinate, i.e. the path of the unimolecular reaction. Thus, it is not necessary to completely close the electronic gap to decompose a molecule. Before the incipient electronic instability occurs, the molecule can decompose. However, if the gap closes completely, the molecule will decompose instantaneously. In the context of SIR, the deformation energy supplied to a molecule is transferred into the electronic system so quickly that the nuclear system cannot respond. Thus, it is necessary to close the gap within $2 t$ after which decomposition of the molecule follows from the resulting mechanical instability.

For a system in equilibrium, where the nuclear degrees of freedom have enough time to follow changes in the electronic energy states, intramolecular mode softening due to applied stress, e.g., pressure, should be observed. Such a phenomenon has been reported, but not sufficiently explained, in studies of pressure effects on the vibrational spectra of liquid nitromethane (another model molecule for sec- ondary explosives) where softening of some normal modes has been detected. ${ }^{39}$ The sensitivity of the critical mode to stress can be estimated from

$$
\frac{\partial \Omega^{2}}{\partial Q}=-\gamma^{2} \frac{\partial \chi_{\mathrm{el}}}{\partial Q}
$$

which is a direct measure of the influence of the stress on the molecular electronic structure. This is important because it shows that by measurement of the strain Grüneisen parameters one can learn about the deformation potential which is related to $\left(\partial \chi_{\mathrm{el}} / \partial Q\right)$.

These considerations about the mechanical instability of a molecule may be generalized to the case of many vibrations. The renormalized normal mode frequencies are found from

$$
D_{i j}-\Omega^{2} \delta_{i j}=0,
$$

where

$$
D_{i j}=\omega^{2} \delta_{i j}-\Gamma_{i} \Gamma_{j} \chi_{\mathrm{el}} .
$$

A similar relation has been used to define molecular compressibility. ${ }^{40}$ The condition for molecular instability can be formulated as $\operatorname{det}(\mathbf{D}) \rightarrow 0$.

From this analysis, it is evident that any defect that creates large local strains will contribute to gap closure and DIMED. There is an extensive literature that posits so-called "hot spots," essentially large local defects and cracks that apparently "nucleate" detonation. These are clearly defects that can generate large internal and local strains that could bring many molecules near the defect very close to the $2 t$ bandwidth necessary to decomposition. Thus, the application of any excess external stress could bring molecules in these regions of the crystal to decomposition much earlier than those that are in defect-free domains.

This analysis of mechanical instability shows that, because of electron-molecular vibration coupling, a molecule will always be unstable to decomposition as a result of HOMO-LUMO gap closure. Since this process is slower than purely electronic processes, but faster than purely vibrational ones, the decomposition is on the time scale of the detonation process.

\section{COOPERATIVITY THROUGH ELECTRON-ELECTRON COUPLING}

So far, we have discussed the process of mechanoelectronic instability of a single molecule in a crystal. However, such molecules actively interact with their surroundings. Thus, we should consider a possible intermolecular chargetransfer contribution to the mechanism of electron delocalization in a SIR. Such a process is very likely because of the large density increases and structural irregularities created at the reaction front. Both effects stimulate intermolecular electron transfer by an increase of the overlap integral arising from stress and molecular inequivalence due to defects, vacancies, etc. The very recent history of hydrogen metallization supports the view that such charge-transfer states play an important role in highly compressed molecular solids. ${ }^{41}$

Consider two molecules in a shock-compressed region. Intermolecular charge-transfer may occur, i.e., a promotion 
of an electron from the HOMO of one molecule (M) to the LUMO of the other. This corresponds to a reaction, M $+\mathrm{M} \Rightarrow \mathrm{M}^{+}+\mathrm{M}^{-}$as presented in Fig. 4 with the assignment of the $E_{R}$ and $E_{P}$ diabatic functions to reactants and products, respectively. The only modification needed to the previous discussion is that the energy of the intermolecular charge transfer be lower than the HOMO-LUMO energy gap for a single molecule since there is energy gain due to Coulomb interactions. This electron-electron coupling, together with lattice relaxation, creates cooperativity through the deformation-induced degeneracy of the electronic states and can be quite efficient in molecular explosives. This problem is left for further study.

\section{CONCLUSIONS}

We have proposed a new microscopic approach to mechanochemical processes. The first step in a shockinduced reaction is the electronic mechanism of molecular decomposition that proceeds by a deformation-induced molecular electronic degeneracy (DIMED) of nonrelaxed HOMO-LUMO states that results in the disappearance of chemical hardness. A qualitative picture for the coupling of electronic and vibrational degrees of freedom has been presented. A result of the analysis is that molecules of high symmetry will be quite unstable to deformation-induced decomposition. A numerical calculation for chemical hardness and of the hardness dependence on normal mode coordinates has been performed for the RDX molecule. The results show that $\mathrm{N}-\mathrm{N}$ bond rupture is a more likely pathway than ring fission. The model further justifies the role of "hot spots" and cracks in enhancing the detonation sensitivity of energetic materials.

By applying our earlier formulation of a theory of solidstate reactivity, ${ }^{8}$ we have shown that the mechanoelectronic mechanism of reactivity is not only possible, but it also allows for a comprehensive picture of the previously suggested HOMO-LUMO gap closure and electromechanical effects. The model has specific applicability to the initial response of energetic materials to shock. In particular, we show that complete closure of the HOMO-LUMO gap is not necessary for a mechanically induced chemical reaction. We are further able to specify the degree of gap closing that is needed to effect such a reaction and to demonstrate the role of symmetry. A natural extension of the single molecule mechanism into a cooperative, intermolecular charge-transfer driven, mechanism is an obvious avenue for further investigation.

Although our focus has been on applying the mechanochemical model to the initial step of a shock-induced reaction, it has application to other outstanding problems. The model developed herein has sufficient generality that it may be expected to provide: the basic concepts for analyzing triboluminescence that does not arise from ambient gas emission, ${ }^{42}$ an approach for understanding conformational polymorphism, ${ }^{43}$ explanation of mechanically induced chemical transformations such as the triclinic phase of anthracene, ${ }^{44}$ and may even be useful in developing new avenues to problems in macromolecular structure. Broadening the paradigm to these problems is underway.

\section{ACKNOWLEDGMENTS}

Support provided by the U. S. Office of Naval Research under Grant No. N00014-98-1-0736 is gratefully acknowledged. T.L. also thanks C.J.E. for the hospitality of his laboratory and the Polish Committee for Scientific Research for travel support. The authors thank both the Theoretical and Computational Chemistry Center at Dalhousie University and the Supercomputing and Networking Center of Wrocław for making their facilities available for the numerical calculations.

${ }^{1}$ L. E. Fried, M. R. Manaa, P. F. Pagoria, and R. L. Thompson, Annu. Rev. Mater. Sci. 31, 291 (2001).

${ }^{2}$ T. Luty, Mol. Phys. Rep. 14, 157 (1996).

${ }^{3}$ A. Tokmakoff, M. D. Fayer, and D. D. Dlott, J. Chem. Phys. 97, 1901 (1993).

${ }^{4}$ W. L. Faust, Science 245, 37 (1989).

${ }^{5}$ H.-S. Im and E. S. Bernstein, J. Chem. Phys. 113, 7911 (2000).

${ }^{6}$ M. M. Kuklja, E. V. Stefanovich, and A. B. Kunz, J. Chem. Phys. 112, 3417 (2000)

${ }^{7}$ J. J. Gilman, Chemistry and Electrochemical Corrosion Stress Corrosion Cracking, Proc. Symp., edited by R. H. Jones (Minerals, Metals and Materials Society, Warrendale, PA, 2001), pp. 3-25; AIP Conf. Proc. 505, 809 (2000); Mater. Res. Soc. Symp. Proc. 539, 145 (1999); Philos. Mag. B 79, 643 (1999); AIP Conf. Proc. 429, 313 (1998); Mater. Res. Soc. Symp. Proc. 453, 227 (1997); Science 274, 65 (1996); AIP Conf. Proc. 370, 215 (1995); Philos. Mag. B 77, 1057 (1995); AIP Conf. Proc. 309, 1349 (1994); J. J. Gilman and R. W. Armstrong, ibid. 309, 199 (1994); J. J. Gilman, Mater. Res. Soc. Symp. Proc. 276, 191 (1992); Philos. Mag. B 67, 207 (1993); J. Mater. Res. 7, 535 (1992).

${ }^{8}$ T. Luty and C. J. Eckhardt, J. Am. Chem. Soc. 117, 2441 (1995).

9 J. J. Gilman, Mater. Res. Innovations 1, 71 (1997); Mater. Res. Soc. Symp. Proc. 410, 199 (1996); Mater. Sci. Eng. 209, 74 (1996).

${ }^{10}$ N. Nagaosa and T. Ogawa, Phys. Rev. B 39, 4472 (1989).

${ }^{11}$ F. J. Owens and J. Sharma, J. Appl. Phys. 51, 1494 (1979).

${ }^{12}$ A. N. Dremin, Philos. Trans. R. Soc. London, Ser. A 339, 355 (1992).

${ }^{13}$ R. G. Pearson, J. Chem. Educ. 64, 561 (1987).

${ }^{14} \mathrm{P}$. Hohenberg and W. Kohn, Phys. Rev. 136, 864 (1964).

${ }^{15}$ R. G. Parr and W. Yang, Density Functional Theory of Atoms and Molecules (Oxford University Press, Oxford, 1989).

${ }^{16}$ A. V. Belik, V. A. Potemkin, and N. S. Zefirov, Dokl. Akad. Nauk SSSR 308, 882 (1989).

${ }^{17}$ P. Ordon and L. Komorowski, Chem. Phys. Lett. 292, 22 (1998).

${ }^{18}$ R. P. Feynman, Phys. Rev. 56, 340 (1939).

${ }^{19}$ R. F. Nalewajski and J. Korchowiec, Charge Sensitivity Approach to Electronic Structure and Chemical Reactivity (World Scientific, Singapore, 1997).

${ }^{20}$ M. H. Cohen, M. V. Ganduglia-Pirovano, and J. Kudrnovsky, J. Chem. Phys. 101, 8988 (1994).

${ }^{21}$ N. O. Lipari, C. B. Duke, and L. Pietronero, J. Chem. Phys. 65, 1165 (1976).

${ }^{22}$ D. Chakraborty, R. P. Muller, S. Dasgupta, and W. A. Goddard II, J. Phys. Chem. A 104, 2261 (2000).

${ }^{23}$ T. R. Botcher and C. A. Wight, J. Phys. Chem. 98, 5441 (1993); 97, 9149 (1993).

${ }^{24}$ J. P. Toscano, Ph.D. dissertation, Yale University, 1993.

${ }^{25}$ M. D. Pace, J. Phys. Chem. 95, 5858 (1991).

${ }^{26}$ M. Choi, H. Kim, and C. J. Chung, J. Phys. Chem. 99, 15785 (1995).

${ }^{27}$ L. R. Rhyzkov and J. M. McBride, J. Am. Chem. Soc. 100, 163 (1996)

${ }^{28}$ X. Zhao, E. J. Hinsta, and Y. T. Lee, J. Chem. Phys. 88, 801 (1988).

${ }^{29}$ C. J. Wu and L. E. Fried, J. Phys. Chem. 101, 8675 (1997).

${ }^{30}$ A. D. Becke, Phys. Rev. A 38, 3098 (1988).

${ }^{31}$ C. Lee, W. Yang, and R. G. Parr, Phys. Rev. B 37, 785 (1988).

${ }^{32}$ M. J. Frisch, G. W. Trucks, H. B. Schlegel et al., Gaussian 94, Revision C.2; Gaussian, Inc., Pittsburgh, PA, 1995

${ }^{33}$ See EPAPS Document No. E-JCPSA6-117-511228 for tables giving the optimized geometry bond lengths and angles for RDX, the atomic Hellman-Feynman forces for a positively and a negatively charged RDX molecule in a Cartesian system, the RDX nuclear hardness and nuclear reactivity indices, the calculated RDX vibrational mode frequencies and their symmetries, and the RDX normal modes that contribute most to the 
molecule's intramolecular deformation energy and hardness renormalization. This document may be retrieved via the EPAPS homepage (http:// www.aip.org/pubservs/epaps.html) or from ftp.aip.org in the directory/ epaps/. See the EPAPS homepage for more information.

${ }^{34}$ B. M. Rice and C. F. Chabalowski, J. Phys. Chem. A 101, 8720 (1997).

${ }^{35}$ R. J. Karpowicz and T. B. Brill, J. Phys. Chem. 88, 348 (1984).

${ }^{36}$ X. Sun, R. L. Fu, K. Yonemitsu, and K. Nasu, Phys. Rev. Lett. 84, 2830 (2000).

${ }^{37}$ M. Peyrard, S. Odiot, E. Oran, J. Boris, and J. Schuur, Phys. Rev. B 33, 2350 (1986).
${ }^{38}$ D. W. Brenner and C. T. White, Phys. Rev. B 39, 1453 (1989).

${ }^{39}$ P. J. Muller, S. Block, and G. J. Piermarini, J. Phys. Chem. 93, 462 (1989).

${ }^{40}$ T. Luty, J. Phys. Soc. Jpn. 61, 3636 (1992).

${ }^{41}$ R. J. Hampley, Z. G. Soos, M. Hanfland, and H.-K. Mao, Nature (London) 369, 384 (1994)

${ }^{42}$ J. I. Zink, Acc. Chem. Res. 11, 289 (1978).

${ }^{43}$ J. Bernstein and J. O. Henck, Cryst. Eng. 1, 67 (1998).

${ }^{44}$ G. M. Parkinson, M. J. Goringe, S. Ramdas, J. O. Williams, and J. M. Thomas, J. Chem. Soc. Chem. Commun. 1978, 134. 